Cahiers d'études italiennes

Pétrarque et le pétrarquisme

\title{
Citations et sources antiques dans le Secretum de Pétrarque (prologue et livre I)
}

\section{Florence Bistagne}

\section{OpenEdition}

\section{Journals}

Édition électronique

URL : http://journals.openedition.org/cei/612

DOI : $10.4000 /$ cei.612

ISSN : 2260-779X

\section{Éditeur}

UGA Éditions/Université Grenoble Alpes

\section{Édition imprimée}

Date de publication : 15 avril 2006

Pagination : 19-32

ISBN : 978-2-84310-081-9

ISSN : $1770-9571$

\section{Référence électronique}

Florence Bistagne, «Citations et sources antiques dans le Secretum de Pétrarque (prologue et livre I) », Cahiers d'études italiennes [En ligne], 4 | 2006, mis en ligne le 15 octobre 2007, consulté le 19 avril 2019. URL : http://journals.openedition.org/cei/612 ; DOI : 10.4000/cei.612 


\title{
CITATIONS ET SOURCES ANTIQUES \\ DANS LE SECRETUM DE PÉTRARQUE (PROLOGUE ET LIVRE I)
}

\author{
Florence Bistagne \\ Université de Provence
}

En apparence la vie de Pétrarque ${ }^{1}$ pourrait sembler extrêmement heureuse: honoré, admiré, couronné poète par la plus prestigieuse des récompenses, il ne connut ni l'exil ni la pauvreté, comme Dante, en ces époques troublées qu'il traversa. Né en 1304, il prend, après de brillantes études à Carpentras, Montpellier et Bologne, le statut ecclésiatique dès 1330 et devient le chapelain de Giovanni Colonna, frère du cardinal Giacomo Colonna. La protection de cette famille, très influente en Italie, en France et en Provence, fut décisive pour Pétrarque. Grâce à eux il fit partie de l'environnement pontifical, connut les intellectuels et hommes politiques importants de son temps et voyagea à travers l'Europe. Le 8 avril 1341 il fut couronné poète au Capitole à Rome, après avoir été l'hôte de Robert d'Anjou roi de Naples, et retourna ensuite à Vaucluse. En 1347 il décide d'abandonner son service auprès des Colonna ${ }^{2}$ et devient chanoine de la cathédrale de Padoue de 1348 à 1351. Sa dernière fonction publique sera au service des Visconti de Milan de 1353 à 1361, pour qui il va en ambassade à Venise, à Prague et à Paris. C'est un homme public donc de premier plan tout à la fois admiré et reconnu comme poète.

Cependant sa vie entière fut tourmentée par le dissidium, par l'instabilité entre tous ces idéaux : vie chrétienne et amoureuse, gloire civile et poétique, vie active et contemplative, mirage du bonheur absolu que l'on

1. Ugo DotTI, Vita di Petrarca, Bari-Rome, Laterza, 1987.

2. Voir Bucolicum carmen VIII: Diuortium, in Francesco PeTrarCA, Rime, Trionfi e poesie latine, éd. G. Martellotti, P.G. Ricci, E. Carrara, E. Bianchi, Milan-Naples, Ricciardi, 1955. 


\section{Florence Bistagne}

trouve dans la poésie et désenchantement lorsqu'on l'affronte. Cette tension est le reflet du contraste entre deux civilisations : celle du Moyen-Âge, centrée sur l'idée de la transcendance et de la justification de la vie terrestre par la vie post mortem, et celle de la Renaissance, dont il est l'un des précurseurs, tendue vers la revalorisation de l'autonomie et de la vie ici bas. Les modèles de Pétrarque sont ainsi d'un côté les Pères de l'Eglise comme saint Augustin ${ }^{3}$, et les grands écrivains latins, Virgile ${ }^{4}$, Cicéron ${ }^{5}$, Tite Live $^{6}$ et Sénèque ${ }^{7}$, qui sont aussi des modèles à imiter pour l'excellence de

3. Pétrarque a possédé notamment un manuscrit du De uera religione, aujourd'hui ms. lat. 2201 de la Bibliothèque nationale de Paris; voir Francisco RICO, "Petrarca y el De uera religione», Italia medioevale e umanistica, XVII, 1974, p.313-364, p.325. Il a également acheté La Cité de Dieu en 1325, et en 1333 Dionigi di Borgo San Sepolcro lui offrit un exemplaire des Confessions.

4. Nous possédons l'exemplaire de Virgile ayant appartenu à Pétrarque, le ms. S. p. Arm. 10, scat. 27 de la Bibliothèque Ambrosienne de Milan dont il existe une édition fac-similé: Francisci Petrarcae Vergilianus Codex ad Publii Vergilii Maronis diem natalem bis millesimum celebrandum quam simillime expressus atque in lucem editus, ed. G. Galbiati - A. Ratti, Milan, Hoepli, 1930. Ce somptueux manuscrit a été enluminé par Simone Martini vers 1340, lors de son séjour à la cour papale d'Avignon à partir de 1336. Le frontispice représente un soldat et un lettré dévoilant un poète couronné de lauriers allongé sous un arbre, la plume à la main. Ces trois personnages sont précédés par un berger et un fermier, allégoriquement représentant les Bucoliques et les Géorgiques. Le soldat représente l'Énéide, le lettré, Servius, commentateur du IV siècle et le poète Virgile. Ces allégories traditionnelles ont été interprétées un peu différemment par Nicolas MANN, dans son Pétrarque, Arles, Actes Sud, 1994 (éd. originale: Petrarch, Oxford, Oxford University Press, 1984), p. 8, où il identifie le poète à Pétrarque lui même, couronné de lauriers depuis 1341 et amant d'une dame au nom précisément de Laure, auteur bucolique (Bucolicum Carmen) et épique (Africa). Sur la feuille de garde du manuscrit en effet figure le récit de sa rencontre avec Laure en Avignon le 6 avril 1327, de la mort de celle-ci et de certains amis. D'autre part les sonnets 77 et 78 du Canzoniere remercient Simone Martini pour un portrait de Laure. Ainsi Pétrarque mêle-t-il, selon Nicholas Mann, ses expériences de commentateur et de poète, d'érudit et de "connaisseur de la peinture contemporaine» (MANN, Pétrarque, cit., p. 9).

5. Leighton D. Reynolds a montré récemment l'importance d'un manuscrit des œuvres philosophiques de Cicéron ayant appartenu à Pétrarque pour la transmission de ces textes: le ms. 9116 de la Bibliothèque Nationale de Madrid. Voir Leighton D. REYNOLDS, "Petrarch and Cicero's Philosophical Works", Les Cahiers de l'Humanisme, I, 2000, p. 37-52. Ce manuscrit de Madrid comprend sept œuvres: De natura deorum, de divinatione, Timaeus, Tusculanae disputationes, Paradoxa, De finibus, Academica posteriora. Silvia Rizzo a identifié, quant à elle, l'exemplaire des Tusculanes ayant appartenu à Pétrarque, le Vitt. Eman. 1632 de la Bibliothèque Nationale de Rome: Silvia RizzO, "Un nuovo codice delle Tusculane dalla biblioteca del Petrarca », Ciceroniana, n.s., vol. IX: Atti del IX Colloquium Tullianum, Courmayeur, 29 apr. - 1 mag. 1995, Rome, 1996, p. 75-104, tav. 1-8. Deux autres manuscrits des Tusculanes de Cicéron sont très connus, celui qui est actuellement à la Bibliothèque Municipale de Troyes, sous le numéro 552, et le ms.. Lat. 5802 de la Bibliothèque Nationale de Paris, où cette œuvre est à la suite de Suétone, Florus, Frontin et Eutrope. Le manuscrit de Troyes comprend également le De Oratore et l'Orator, qui est défini comme le quatrième livre du De Oratore. La plus grande découverte de Pétrarque fut celle des Lettres à Atticus en 1345 dans la librairie du Chapître de Vérone. Voir dans les Familiares les nombreuses lettres où il l'évoque, et notamment XXII, 2, adressée à Boccace en 1359 (in Francesco Petrarca, Opere, a cura di Mario Martelli, Florence, Sansoni, 1992).

6. Nous ne nous étendrons pas sur la transmission du texte de Tite-Live, mais nous bornons à rappeler que Pétrarque en est un maillon essentiel de la chaîne. Voir Giuseppe BILLANOvich, La tradizione del testo di Livio e le origini dell'umanesimo, II: Il Livio del Petrarca e del Valla, Padoue, 
leur langue latine, de l'autre. Pétrarque fut ainsi le fondateur du renouveau de l'intérêt pour les auteurs antiques, leur langue, leur vision du monde, en débarassant les textes des scories de la transmission. Dans une lettre à Boccace en 1359 (Familiares XXII, 2), il parle de la façon dont les œuvres de ses écrivains préférés se sont gravées dans sa mémoire à tel point qu'il en oublie parfois qui les a écrites et même si c'est quelqu'un d'autre que lui qui les a écrites... Ce phénomène d'innutrition totale est une vraie théorie poétique, et signale sa préférence pour les auteurs antiques, même si elle ne saurait être avouée.

Le Secretum ${ }^{8}$ s'insère tout à fait dans ce cadre: dialogue à l'antique sur le modèle de Cicéron, confession intime sur le modèle de saint Augustin, présence de ce dernier en miroir dans l'œuvre autobiographique, conflit entre les deux figures de l'âme du poète alors mises en scène, Franciscus et Augustinus. L'œuvre est divisée en trois livres, comme le De Oratore de Cicéron, chacun retransmettant une journée de conversation en présence de la Vérité. À la fin du combat Franciscus ne sera pas tout à fait vaincu par le sévère Augustinus, et son adversaire sera obligé lui aussi de reconnaître sa propre dette envers les auteurs antiques.

Et nequid tibi subtraham, scito me, ut opus illud inciperem, unum maxime Ciceronis tui verbum induxisse. Affuit Deus incepto, ut ex paucis seminibus messis opima consurgeret ${ }^{9}$.

Nous allons étudier comment ce combat s'exprime dans la préface et le livre I par l'usage des auteurs antiques, explicitement et implicitement, en examinant le vocabulaire des deux protagonistes.

Antenore, 1981, qui présente et fait l'exégèse du ms. Harley 2493 de la British Library à Londres ayant appartenu à Pétrarque puis à Lorenzo Valla.

7. Il n'existe pas d'inventaire complet des livres ayant appartenu à Pétrarque, mais on peut penser qu'il posséda les Lettres à Lucilius, qui lui ont fourni un modèle pour la structure des Familiares, divisée en vingt-quatre livres. Il connaît parfaitement les œuvres de Sénèque bien sûr, et plusieurs annotations dans divers manuscrits de Cicéron portent des renvois à des passages de Sénèque, par exemple dans le manuscrit de Madrid étudié par L.D. Reynolds, en marge du commentaire aux Tusculanes I, 3-6.

8. Le Secretum est un dialogue philosophique autobiographique, écrit entre 1347 et 1353 et dont l'action se situe en 1342-1343, moment de crise existentielle pour Pétrarque. Une controverse sur la date de composition de l'ouvrage par rapport à la date de l'action a été résolue par les études de Hans Baron et de Francisco Rico. Ils ont montré que la rédaction de cette œuvre n'est pas antérieure à celle du De Otio religioso, écrit après l'entrée de son frère Gherardo dans l'ordre des Chartreux. Voir Hans BARON, Petrarch's Secretum, Cambridge (Mass.), The Medieval Academy of America, 1985; Francisco Rico, «Sobre la cronologia del Secretum. Las viejas leyendas y el fantasma nuevo de un lapsus biblico", Studi Petrarcheschi, n.s., I, 1984, p. 51-102.

9. Francesco Petrarca, Secretum, a cura di Enrico Fenzi, Milan, Mursia, 1992, p. 138: «et, pour ne rien te cacher, sache que pour commencer cette œuvre ce fut avant tout un seul mot de ton cher Cicéron qui m’y poussa. Dieu m'aida au départ en sorte que de ces modestes semences est née une riche moisson". Nous traduisons. 


\section{Florence Bistagne}

Dès le prologue en effet, au milieu d'une vision de type médiéval ${ }^{10}$, le concept vient de Sénèque, le vocabulaire de Virgile et la forme de Cicéron ${ }^{11}$ :

Attonito michi quidem et sepissime cogitanti ${ }^{12}$ qualiter in hanc uitam intrassem, qualiter ve forem egressurus ${ }^{13}$, contigit nuper ut non, sicut egros animos solet, somnus opprimeret, sed anxium atque pervigilem mulier quedam inenarrabilis etatis et luminis, formaque non satis ab hominibus intellecta, incertum quibus viis adiisse videretur. Virginem tamen et habitus nuntiabat et facies ${ }^{14}$.

Franciscus ne peut d'ailleurs que s'adresser à elle par des vers de Virgile, et cela la fera se nommer. C'est l'intervention du poète antique qui donne corps à l'allégorie médiévale humainement inconcevable:

O quam te memorem, uirgo? namque haud tibi vultus

Mortalis, nec vox hominem sonat ${ }^{15}$

Pétrarque procède donc à la fois par citations explicites et par renvois ${ }^{16}$. Nous avons dénombré les deux types de sources, et nous verrons comment la précellence des auteurs antiques se manifeste dans ce prologue et

10. Voir par exemple DanTE, Enfer II, 56-57, l'apparition de Béatrice descendue dans les limbes, ou encore Vita Nuova III, apparition d'Amour.

11. Secretum, ed. cit., p. 94: «Récemment, absorbé dans mes pensées et méditant, comme bien souvent, sur la façon dont j'étais entré dans cette vie et sur la façon dont j'allais la quitter, vint à moi, non frappé par le sommeil, comme il arrive aux esprits malades, mais angoissé et tout à fait éveillé, une femme d'un âge et d'une splendeur indicibles, et d'une beauté à peine intelligible pour les hommes, et je ne savais pas par quelles voies elle était arrivée jusqu'ici. Son apparence pourtant et son visage annonçaient une vierge». Nous traduisons.

12. Voir Cicéron, De Oratore I, 1 : «cogitanti mihi saepenumero [... ]»; " comme je médite souvent $[\ldots]$ ". Nous traduisons.

13. Voir SÉNEQUE, Lettres à Lucilius XXII, 14 : «nemo non ita exit e uita tanquam modo intraverit "; " personne ne quitte la vie de la façon dont il y est entré». Nous traduisons.

14. Voir Virgile, Énéide I, 315: "virginis os habitumque gerens»; "montrant le visage et l'apparence d'une vierge".

15. VIRGILE, Énéide I, 327-328: «comment t'appeler, Vierge? ni ton visage en effet/n'est d'une mortelle, et le son de ta voix n'est pas d'humain ". Nous traduisons.

16. Par citation explicite il faut entendre la présence exacte d'un auteur antique à laquelle Pétrarque lui-même donne ce statut de citation, et par renvoi une source antique non dévoilée au lecteur, mais implicitement reconnue par ce dernier s'il a le même horizon culturel. En effet, ce ne sont pas des citations retouchées, mais bien plutôt des réutilisations de vocabulaire identique tellement mêlées au texte de Pétrarque qu'on les discerne à peine. Utiliser les expressions spumantia labra et luridi dentes (Secretum I, 58) pour évoquer le corps mort, par exemple, renvoie nécessairement à Horace et à Virgile sans être une citation explicite, de même l'expression summis auribus à Virgile. Pour faire une comparaison: ce sont presque des épithètes homériques. Sur ces définitions, voir Identifier sources et citations (L'atelier du Médiéviste), ed. Jacques Berlioz et alii, Turnhout, Brepols, 1994, p. 5 suiv., où une catégorie supplémentaire est envisagée, le démarquage, "copie pure et simple sans citation. Aujourd'hui appelé plagiat, il est monnaie courante au Moyen-Âge» (p.6). Nous utilisons cette terminologie, en rappelant également que tout texte présent dans un autre texte est dit "source». 
le livre I, même dans la bouche d'Augustinus. Il faut noter que Pétrarque ne donne jamais le nom des œuvres d'où il tire la citation sauf pour les Tusculanes de Cicéron, qui est l'œuvre qu'Augustinus reconnaît comme étant à la base de sa propre réflexion. Il est donc une caution supplémentaire indiscutable. Celui-ci le cite même par erreur à la place de Sénèque ${ }^{17}$, alors que nous verrons qu'en ce qui concerne les renvois implicites, Sénèque est l'auteur favori d'Augustinus.

Les citations explicites:

\begin{tabular}{|c|c|c|}
\hline AutEur CitÉ & Euvre & LOCUTEUR \\
\hline Virgile & Énéide I, 327-328 ${ }^{18}$ & Franciscus \\
\hline Cicéron & De Amicitia I, $3^{19}$ & Franciscus \\
\hline Cicéron & Tusculanes V, $12,35^{20}$ & Augustinus \\
\hline Virgile & Énéide IX, 64121 & Augustinus \\
\hline Virgile & Énéide IV, $449^{22}$ & Augustinus \\
\hline Ovide & Pontiques III, 1, $35^{23}$ & Augustinus \\
\hline Horace & Épîtres I, 18, 82-85 24 & Augustinus \\
\hline Virgile & Enéide VI, 365 ; 370-371 25 & Franciscus \\
\hline Sénèque & Lettres à Lucilius I, 226 & Augustinus \\
\hline Virgile & Énéide X, $549^{27}$ & Augustinus \\
\hline Virgile & Énéide V, $849^{28}$ & Franciscus \\
\hline
\end{tabular}

17. Secretum, ed. cit. p. 132.

18. Voir note 15 ; cf. Secretum, Proemium.

19. "Inquam et inquit sepius interponerentur, atque ut coram res agi velut a presentibus videretur»; " [afin de] de ne pas trop souvent intercaler 'dis-je'ou 'dit-il', et que la scène semble se passer comme entre des personnes présentes" (nous traduisons); cf. Secretum, Proemium.

20. "Sola uirtus animum felicitat", "seule la vertu rend l'âme heureuse"; Secretum I, 34.

21. "Macte nova virtute puer: sic itur ad astra"; "honneur à ce courage nouveau, jeune homme: c'est ainsi que l'on va jusqu'aux cieux" (nous traduisons); cf. Secretum I, 34.

22. "Mens immota manet, lacrime volvuntur inanes"; "son âme reste inébranlée, ses larmes coulent en vain" (nous traduisons); cf. Secretum I, 42.

23. "Velle parum est; cupias, ut re potiaris, oportet»; "vouloir est peu de choses; c'est désirer qu'il faut pour te rendre maître de ton objet» (nous traduisons); cf. Secretum I, 44.

24. "Ad te post paulum ventura pericula cernis"; "tu vois les dangers qui vont venir jusqu'à toi peu après" (nous traduisons); cf. Secretum I, 50.

25. "Eripe me his, invicte, malis [...] / Da dextrum misero, et tecum me tolle per undas/sedibus ut saltem placidis in morte quiescam ", "tire moi de ces maux, invincible [...] / tends la main à un malheureux et emmène moi avec toi par les ondes/afin qu'au moins dans la mort je repose en un lieu de paix" (traduction de Jacques Perret (VIrgILE, Énéide, texte établi et trad. par J. Perret, tome II : Livres V-VIII, Paris, Les Belles Lettres, 1993) légèrement modifiée); cf. Secretum I, 58.

26. "In hoc fallimur quod mortem prospicimus"; "nous nous trompons en voyant la mort au loin» (nous traduisons); cf. Secretum I, 60.

27. "Canitiemque sibi et longos promiserat annos"; "il s'était promis des cheveux blancs et de longues années» (nous traduisons); cf. Secretum I, 60.

28. "Me ne huic confidere monstro"; "de ne pas me fier à ce miracle» (traduction de Jacques Perret, cit.) ; cf. Secretum I, 62. 


$\begin{array}{lll}\text { Horace } & \text { Odes I, 11, 7-8 } 29 & \text { Franciscus } \\ \text { Ovide } & \text { Amours II, 11, 15 } & \text { Franciscus } \\ \text { Virgile } & \text { Énéide VI, 730-734 } 30 & \text { Augustinus } \\ \text { Cicéron } & \text { Tusculanes I, 16, 37-38 } & \text { Augustinus }\end{array}$

À la lecture de ce tableau, l'on peut mettre en évidence plusieurs faits:

Virgile, le poète païen, est l'auteur le plus cité, ce qui tend à conforter l'identification entre Pétrarque et ce dernier, surtout après le cournonnement de 1341. La seule œuvre citée est l'Énéide, le grand poème épique, modèle de l'Africa pour Pétrarque.

Augustinus est le personnage qui donne le plus de citations d'auteurs antiques, il ne saurait donc se défaire de leur influence lui non plus. Dans le prologue et le livre I il est celui qui parle le plus et questionne Franciscus. Il a donc dans sa maieutique recours aux auteurs latins.

De plus, si l'on considère l'identification qui a souvent été faite d'Augustinus comme sage stoïcien, outre le fait d'être un moraliste chrétien, force est de voir qu'elle ne saurait être vérifiée dans les rappels explicites de Sénèque. En effet ce dernier est cité une seule fois; le lecteur moderne peut retrouver un passage de Sénèque (Lettres à Lucilius I, 2) mais que Augustinus attribue par erreur à Cicéron. Sénèque n’apparaît donc pas du tout.

Le corpus des auteurs cités est exactement le même pour les deux personnages: Virgile, Cicéron, Ovide et Horace. Les graphiques ci dessous nous montrent qu'il n'y a absolument pas de prépondérance d'auteurs moralistes, mais bien des poètes.

29. «Loquimur dum talia, forsan/innumeris properata uiis, in limine mors est» «pendant que nous tenons de tels propos/la mort s'est hâtée par d'innombrables chemins, et elle est déjà sur le seuil». Cette citation est une contamination de deux vers, l'un d'Horace, «dum loquimur, fugerit invida/aetas" (Odes I, 11, 7-8), et l'autre d'Ovide, "dum loquor hora fugit» (Amours II, 11, 15); cf. Secretum I, 62.

30 . «Igneus est illis vigor et celestis origo/seminibus, quantum non noxia corpora tardant/terrenique hebetant artus, moribundaque membra./Hinc metuunt cupiuntque dolent gaudentque, neque auras/respiciunt, clause tenebris et carcere ceco"; "une vigueur de feu, une empreinte céleste paraissent en eux,/du fait de ces germes, pour autant qu'ils ne sont pas appesantis par l'impureté de la matière,/émoussés par des organes faits de terre et des membres voués à la mort./Dès lors craintes et désirs pour nos âmes, chagrins et joies, et les souffles/du ciel elle ne les sentent plus, encloses de ténèbres en aveugle prison" (traduction de Jacques Perret, cit.); cf. Secretum I, 64.

31. «Nichil animo videre poterant, ad oculos omnia referebant; magni autem est ingenii revocare mentem a sensibus et cogitationem a consuetudine abducere»; "ils ne pouvaient rien voir avec l'esprit et rapportaient tout aux yeux; or un esprit supérieur détourne sa réflexion des sens et éloigne sa pensée de la coutume» (nous traduisons); cf. Secretum I, 66. 
Virgile est cité par les deux personnages pour environ la moitié de leur citations. Pour Augustinus, il est immédiatement suivi par Cicéron, encore deux foi plus cité que les deux poètes, à présence égale, Ovide et Horace. En revanche pour Franciscus, Ovide, Horace et Cicéron sont au même niveau, et chacun trois fois moins important que Virgile.

$\begin{array}{lll}\text { Auteur } & \text { Euvre } & \text { Nicéron } \\ & \text { De Amicitia, I, 3 } & 3 \\ & \text { Tusculanes I, 16, 37-38 } & 1 \\ & \text { Tusculanes V, 12, 35 } & 1 \\ \text { Horace } & & 2 \\ & \text { Épîtres I, 18, 82-85 } & 1 \\ \text { Ovide } & \text { Odes I, 11, 7-8 } & 1 \\ & & 2 \\ \text { Sénèque } & \text { Amours II, 11, 15 } & 1 \\ & \text { Pontiques III, 1, 35 } & 1 \\ \text { Virgile } & & 1 \\ & \text { Lettres à Lucilius I, 2 } & 1 \\ & & 7 \\ & \text { Énéide I, 327-328 } & 1 \\ \text { Énéide IV, 449 } & 1 \\ \text { Énéide V, 849 } & 1 \\ & \text { Énéide VI, 365; 370-371 } & 1 \\ & \text { Énéide VI, 730-734 } & 1 \\ \text { Énéide X, 549 } & 1 \\ \text { Énéide IX, 641 } & 1 \\ & & 1\end{array}$

LOCUTEUR

AUTEur

$\mathrm{NB}$

Augustinus

Cicéron

9

Horace

Ovide

Sénèque

Virgile

NB

1

1

.




\section{Florence Bistagne}

\section{Les renvois}

En étudiant le texte de Pétrarque nous pouvons souvent retrouver des échos de la littérature antique, aussi bien sur le plan des concepts, que sur le plan lexical ${ }^{32}$. Nous avons répertorié ci-dessous les passages sur lesquels Pétrarque s'appuie, en essayant d'être exhaustif ${ }^{33}$. Bien entendu la liste est ouverte et pourra alors donner lieu à modification des statistiques que nous en tirons.

\begin{tabular}{|c|c|c|}
\hline SOURCE & EUvre & LOCUTEUR \\
\hline Cicéron & De Oratore, proemium & Franciscus \\
\hline Sénèque & $\begin{array}{l}\text { Lettres à Lucilius } \\
\text { XXII, } 14\end{array}$ & Franciscus \\
\hline Virgile & Énéide I, 315 & Franciscus \\
\hline Horace & Odes II, 2, 2 & $\begin{array}{l}\text { La vérité } \\
\text { canendo» } 37\end{array}$ \\
\hline \multicolumn{2}{|c|}{ Bucoliques II, 24} & La vérité \\
\hline Sénèque & Lettres à Lucilius VI, 1-2 & Franciscus \\
\hline Cicéron & Tusculanes III, 10, 23 & Franciscus \\
\hline
\end{tabular}

\author{
RENVOI \\ "Cogitanti mihi saepenumero " 34 \\ "Nemo non ita exit e uita tanquam \\ modo intraverit" 35 \\ "Virginis os habitumque gerens» 36 \\ «Movit Amphion lapides \\ "Amphion dircaeus» 38 Virgile \\ "Quibusdam aegris gratulatio fit cum \\ ipsi aegros se esse senserunt" 39 \\ «Ut medici causa morbi inventa curatio \\ nem inventam putant, sic nos causa
}

32. Voir par exemple comment Augustinus s'adresse à la Vérité: «tu michi dux, tu consultrix, tu domina, tu magistra ", et le prologue du livre V des Tusculanes, adressé à la Philosophie: "o uitae philosophia dux, o uirtutis indagatrix expultrixque uitiorum ": même harmonie des membres de phrases, même utilisation des mots en $-i x$. Pétrarque a aussi sans doute en mémoire l'apostrophe de Dante, Enfer II, 140: "Tu duca, tu segnore e tu maestro», mais la source est plus ancienne et commune. Voir également notes 12 à 14 .

33. L'édition commentée par Enrico Fenzi (op. cit.) donne de nombreuses sources, mais surtout en ce qui concerne les concepts repris par Pétrarque aux auteurs stoiciens et à saint Augustin lui-même, ainsi que de nombreux renvois aux autres œuvres de Pétrarque. Nous nous sommes appuyés sur cette édition, mais nous attachons davantage au vocabulaire. Ainsi nous avons identifié de simples expressions reprises à la poésie élégiaque par exemple (Properce, vitium dilue, etc.) et non relevées par la critique comme signifiantes. Les outils essentiels pour ces identifications, outre la fréquentation assidue des textes, et surtout permettant de vérifier les intuitions, sont les concordances, et la Aureae Latinitatis Bibliotheca, Zanichelli, Bologne (CDRom).

34. "Comme je médite souvent"; cf. Secretum, Proem.: "attonito michi quidem et sepissime cogitanti»; "absorbé dans mes pensées et méditant, comme bien souvent".

35. "Personne ne quitte la vie de la façon dont il y est entré»; cf. Secretum, Proem.: "qualiter in hanc uitam intrassem, qualiter ve forem egressurus »; «sur la façon dont j'étais entré dans cette vie et sur la façon dont j'allais la quitter".

36. «Montrant le visage et l'apparence d'une vierge»; cf. Secretum, Proem.: «virginem tamen et habitus nuntiabat et facies»; "son apparence pourtant et son visage annonçaient une vierge».

37. "Amphion fit bouger les rochers par sa poésie»; cf. Secretum, Proem.: "Amphion ille dircaeus [...] mirabili artificio ac poeticis, ut proprie dicam, manibus erexisti»; "(tel) le fameux Thébain Amphion, par ton art admirable et, pour parler juste, avec tes mains de poètes, tu as érigé $[\ldots] »$.

38. "Amphion, le Thébain"; cf. Secretum, Proem., loc. cit.

39. "C'est une joie pour certains malades, lorsqu'ils se sont rendus compte eux mêmes qu'ils étaient malades»; cf. Secretum I, 24: "eo propinquior morti est quo eger ipse a proprii morbi cognitione remotior "; «maladie d'autant plus près d'être mortelle que le malade lui même est loin de comprendre son propre mal!». 
Citations et sources antiques dans le Secretum de Pétrarque

\begin{tabular}{|c|c|c|c|}
\hline & & & $\begin{array}{l}\text { aegritudinis reperta medendi facultatem } \\
\text { reperiemus» } 40\end{array}$ \\
\hline Sénèque & $\begin{array}{l}\text { Lettres à Lucilius } \\
\text { LXX, } 18\end{array}$ & Augustinus & $\begin{array}{l}\text { "Nullius rei meditatio tam necessaria } \\
\text { est» } 41\end{array}$ \\
\hline Cicéron & De senectute XIX, 67 & Augustinus & «Impendens periculum» 42 \\
\hline Sénèque & Lettres à Lucilius LXVII, 4 & Augustinus & $\begin{array}{l}\text { "Non sum tam demens ut aegrotare } \\
\text { cupiam» } 43\end{array}$ \\
\hline Cicéron & Tusculanes $\mathrm{V}, 8,23$ & Franciscus & $\begin{array}{l}\text { "Qui beatus est, non intellego quid } \\
\text { requirat, ut sit beatior» } 44\end{array}$ \\
\hline Cicéron & Tusculanes $\mathrm{V}, 10,29$ & Franciscus & $\begin{array}{l}\text { «Equidem eos existimo, qui sint in } \\
\text { bonis nullo adiuncto malo...» } 45\end{array}$ \\
\hline Cicéron & Tusculanes V, 29, 83 & Franciscus & $\begin{array}{l}\text { "Necesse est semper beatum esse sapien } \\
\text { tem» } 46\end{array}$ \\
\hline Cicéron & Tusculanes V, 9, 25 & Franciscus & "Tertium hoc sit in potestate fortunae" 47 \\
\hline Sénèque & Lettres à Lucilius VII, 12 & Augustinus & $\begin{array}{l}\text { «Ista, mi Lucili, condenda in animo } \\
\text { sunt, ut contemnas voluptatem ex plu } \\
\text { rium adsensione venientem» } 48\end{array}$ \\
\hline Sénèque & Lettres à Lucilius LXX, 15 & Franciscus & «Stoicorum sententia» 49 \\
\hline
\end{tabular}

40. «Tout comme les médecins pensent que, lorsqu'on a trouvé la cause de la maladie, on a trouvé le remède, de même nous, lorsque nous aurons trouvé la cause de la maladie, nous trouverons la façon de la soigner"; cf. Secretum I, 24, loc. cit.

41. «La méditation sur aucun autre sujet n'est aussi nécessaire»; cf. Secretum I, 28: «nichil efficacius reperiri quam memoriam proprie miserie et meditationem mortis assiduam»; "on ne trouve rien de plus efficace que de se rappeler constamment ses propres malheurs et de méditer sur la mort".

42. "Le danger imminent"; cf. Secretum I, 28 : «et impendens periculum dissimulatis agnoscere "; "(je m'étonne que) vous fassiez semblant de ne pas reconnaître que le danger est imminent".

43. «Je ne suis pas si fou que je souhaite être malade»; cf. Secretum I, 28 : «putas ne quempiam adeo delirare ut morbo ancipiti correptus non summe cupiat sanitatem?»; "penses-tu que je délire à ce point que, si j'étais atteint d'une dangereuse maladie, je ne désirerais pas avant tout retrouver la santé?».

44. "Je ne comprends pas ce qu'il faut à celui qui est heureux pour être plus heureux»; cf. Secretum I, 30: «siquidem et bonorum cumulatissimus acervus felices facit, et quicquid inde decesserit pro ea parte necesse est efficiat infelices "; "si certes un tas de biens accumulés nous rend heureux, il en découle nécessairement que tout ce qui vient à en manquer nous rend, pour sa part, malheureux». Dans cette phrase Pétrarque emploie aussi le mot acervus, que Cicéron emploie dans les Tusculanes $\mathrm{V}, 45$, au sens propre de "monceau", "tas (de grains)».

45. "Quant à moi je pense que ceux qui le sont (NdT. heureux) sont ceux qui se trouvent au milieu des biens sans aucun malheur qui vient s'y ajouter»; cf. Secretum I, 32: «dubitari igitur meo iudicio non potest quin multi quidem inviti nolentesque sint miseri»; «à mon avis donc on ne peut douter que beaucoup sont malheureux tout à fait malgré eux et sans le vouloir ».

46. «Le sage est nécessairement heureux»; cf. Secretum I, 32 loc. cit.

47. «La troisième est du ressort de la fortune»; cf. Secretum I, 32: "quod prima duo nostri arbitrii, tertium hoc sit in potestate fortune"; "parce que les deux premières choses dépendent de notre volonté, la troisième est du ressort de la fortune».

48. «Tu dois ancrer, mon cher Lucilius, dans ton esprit, de mépriser le plaisir approprié au goût de la masse"; cf. Secretum I, 34: "o te omnium infelicem si ad ueritatis inquisitionem per vulgi deliramenta contendis "; " $\mathrm{O}$, malheureux entre tous, si tu fondes ta quête de la vérité au moyen des délires de la foule».

49. "Selon l'avis des Stoïciens»; cf. Secretum I, 34 : «ad stoiocorum precepta me revocas»; « et stoicorum sententias publicis erroribus preferendas esse non dubito"; "tu me renvoies aux préceptes des Stoïciens»; "et je ne doute pas que l'on doive préférer les opinions des Stoïciens aux erreurs communes». 


\section{FLORENCE BISTAGNE}

\begin{tabular}{|c|c|c|c|}
\hline Cicéron & De finibus III, 7, 26 & Franciscus & $\begin{array}{l}\text { "Omnes sapientes semper feliciter, abso } \\
\text { lute, fortunate vivere, nulla re impediri, } \\
\text { nulla prohiberi, nulla egere" } 50\end{array}$ \\
\hline Cicéron & Tusculanes IV, 17, 37 & Franciscus & $\begin{array}{l}\text { "Ei nihil inprovisum accidere possit, } \\
\text { nihil inopinatum, nihil omnino } \\
\text { novum» } 51\end{array}$ \\
\hline Properce & III, 17,6 & Franciscus & $\begin{array}{l}\text { "Tu vitium ex animo dilue, Bacche, } \\
\text { meo } 52\end{array}$ \\
\hline Sénèque & Lettres à Lucilius CXVI, 8 & Augustinus & $\begin{array}{l}\text { "Nolle in causa est, non posse praetendi } \\
\text { tur» } 53\end{array}$ \\
\hline Virgile & Enéide VI, 29 & Augustinus & $\begin{array}{l}\text { «Daedalus ipse dolos tecti ambagesque } \\
\text { resolvit» } 54 \text {. }\end{array}$ \\
\hline Cicéron & De senectute XIX, 71 & Augustinus & $\begin{array}{l}\text { "Aliquandoque in portum ex longa navi } \\
\text { gatione esse venturus" } 55\end{array}$ \\
\hline Sénèque & Lettres à Lucilius XCII, 10 & Augustinus & "Prima pars hominis est ipsa uirtus" 56 \\
\hline Horace & Odes I, 22, 1 & Franciscus & «Integer vitae scelerisque purus» 57 \\
\hline Sénèque & Lettres à Lucilius LXVII, 4 & Augustinus & «Non sum tam demens ut $[\ldots] » 58$ \\
\hline Sénèque & Lettres à Lucilius LXXVII, 11 & Augustinus & $\begin{array}{l}\text { "Nemo tam imperitus est ut nesciat } \\
\text { quandoque moriendum" } 59\end{array}$ \\
\hline Sénèque & Lettres à Lucilius LIX, 12 & Augustinus & «Omnes me filium Jovis dicunt sed \\
\hline
\end{tabular}

50. «Tous les sages vivent toujours dans la félicité, dans la perfection, dans le bonheur, rien ne les entrave, rien ne leur est interdit, ils ne manquent de rien»; cf. Secretum I, 34: «quam ob rem, stante licet stoicorum sententia, tolerari potest multos invitos ac dolentes optantesque contrarium esse miserrimos"; "c'est pourquoi, bien que l'opinion des Stoïciens reste valable, on peut accepter que beaucoup soient très malheureux malgré eux, et en souffrent et souhaitent le contraire».

51. «Et rien ne peut lui arriver qu'il n'ait pas prévu, rien auquel il n'ait pensé, rien d'absolument inoui»; cf. Secretum I, 34 loc. cit.

52. «Toi, Bacchus, dissipe ce vice de mon âme»; cf. Secretum I, 36: "quantisque cum lacrimis sordes meas diluere nisus sim»; " (tu peux dire) et avec combien de larmes j’ai tenté de dissiper mes souillures".

53. «C'est le manque de volonté qui est en cause, et on invoque l'incapacité»; cf. Secretum I, 40 : «verba vero, quibus uti te velim, hec sunt: ut ubi «ultra te non posse», dixisti « ultra te nolle» fatearis»; "mais voici les mots que je voudrais que tu utilises: quand tu as dit «je ne peux pas aller plus loin», avoue «je ne veux pas aller plus loin»».

54. «Dédale lui-même dénoue les artifices et les entrelacs du palais» (traduction Jacques Perret modifiée); cf. Secretum I, 40 : «nec tamen admiror te in his nunc ambagibus obvolutum in quibus olim ego ipse iactatus »; «je ne m'étonne cependant pas que tu sois à présent pris dans les entrelacs où je fus moi-même jadis ballotté».

55. "J'arriverai enfin au port après une longue navigation"; cf. Secretum I, 42: "tandem aliquando in portum ex tam multis tempestatibus revertenti»; «revenant enfin une bonne fois au port après tant de tempêtes".

56. "La partie la plus importante de l'homme est cette vertu»; cf. Secretum I, 46: « ad hoc ipsum per se virtutis desiderium pars est magna virtutis»; "en outre le désir en soi de la vertu est une grande partie de la vertu".

57. «Cette vie intacte, vierge de toute faute»; cf. Secretum I, 48 : "quid igitur censes esse faciendum, ut integer animus, discussis terre compedibus, tollatur ad supera?»; "que penses tu que l'on doive faire pour que l'âme s'élève intacte, après avoir brisé ses entraves terrestres, vers les choses supérieures?».

58. «Je ne suis pas si dément que [...]»; cf. Secretum I, 48: «nemo tam demens est, nisi sit idem prorsus insanus, cui $[\ldots]$ »; « personne n'est si dément, à moins d'être en même temps absolument fou, pour $[\ldots] »$.

59. "Personne n'est si ignorant au point de ne pas savoir qu'on doit mourir un jour»; cf. Secretum I, 48: «nemo tam demens est [...] cui non interdum conditio proprie fragilitatis occurrat; qui non, si interrogetur, respondeat se esse mortalem et caducum habitare corpusculum "; 
Citations et sources antiques dans le SeCretum de Pétrarque

\begin{tabular}{|c|c|c|c|}
\hline Ovide & Tristes I, 11, 23 & Augustinus & $\begin{array}{l}\text { dolor hic hominem me esse testatur" } 60 \\
\text { "Quocumque adspicio, nihil est nisi } \\
\text { mortis imago" } 61\end{array}$ \\
\hline Stace & Thébaïde, XI, 252 & Augustinus & «Summis auribus» 62 \\
\hline Horace & Odes IV, $13,10-11$ & Augustinus & «Luridi dentes» 63 \\
\hline Virgile & Énéide I, 324 & Augustinus & "Spumantia membra» 64 \\
\hline Sénèque & Lettres à Lucilius VI, 6 & Augustinus & $\begin{array}{l}\text { "Homines amplius oculis quam auribus } \\
\text { credunt } 65\end{array}$ \\
\hline Cicéron & De senectute XX, 74 & Augustinus & $\begin{array}{l}\text { "Moriendum enim certe est, et incertum } \\
\text { an hoc ipso die" } 66\end{array}$ \\
\hline Virgile & Énéide VI, 273-296 & Augustinus & «Ultrices curae», «pallentes morbi» 67 \\
\hline Horace & Odes III, 10, 10 & Augustinus & "Graviore casu " $68^{1}$ \\
\hline Ovide & Tristes III, 4, 16 & Franciscus & "Cymba rimosa " 69 \\
\hline Virgile & Énéide V, 125-6 & Franciscus & $\begin{array}{l}\text { "Quod tumidis submersum tunditur } \\
\text { olim/fluctibus" } 70\end{array}$ \\
\hline Sénèque & Lettres à Lucilius XIX, 2 & Franciscus & $\begin{array}{l}\text { "In freto viximus, moriamur in } \\
\text { portu» } 71\end{array}$ \\
\hline
\end{tabular}

"personne n'est si dément $[\ldots]$ pour ne pas avoir parfois à l'esprit sa condition et sa propre fragilité; pour, si on l'interroge, ne pas répondre, qu'il est mortel et qu'il habite un tout petit corps qui va passer".

60. "Tous disent que je suis le fils de Jupiter, mais cette douleur témoigne que je suis un homme»; cf. Secretum I, 48: «id enim et membrorum dolor et febrium tentamenta testantur»; "en effet la douleur des membres et les épreuves des fièvres en témoignent".

61. «Partout où mes regards se portent, il n'y a rien si ce n'est l'image de la mort»; cf. Secretum I, 50: «quibus undique circumsepti non potestis oculos advertere, ubi non eis occurrat proprie mortalitatis effigies"; " et vous qui êtes cernés de toutes parts, vous ne pouvez tourner vos regards nulle part où ne se présente à eux le spectre de votre propre mort»; effigies est le mot propre qui désigne le spectre, le fantôme, et qui est employé ailleurs par Ovide (Métamorphoses XIV, 358).

62. "D'une oreille distraite»; cf. Secretum I, 54: "non tamen vel sillaba hec summis auribus excepta vel rei ipsius recordatio compendiosa sufficiet"; "cependant il ne suffit pas que l'on recueille d'une oreille distraite ces syllabes ou le souvenir sommaire de ce qu'elles veulent dire».

63. «Des dents jaunâtres»; cf. Secretum I, 54: «ad hec [...] luridos dentes»; «en outre [...] des dents jaunâtres".

64. «Des membres écumants»; cf. Secretum I, 54 : «spumantia labra»; «des lèvres écumantes».

65. "Les hommes font bien plus confiance à leurs yeux qu’à leurs oreilles»; Secretum I, 54 : "tenacior enim esse solet visorum quam auditorum recordatio"; «le souvenir de ce que l'on a vu est d'ordinaire plus persistant que celui que l'on a entendu ".

66. «Car nous devons mourir, cela est certain, quel sera le jour, cela est incertain »; Secretum I, 56: «nil morte certius, nil hora mortis incertius »; « rien de plus certain que la mort, rien de plus incertain que l'heure de cette mort".

67. «Les soucis vengeurs», «les maladies livides»; Secretum I, 56: «ultrices furiae», "pallentis Orchi »; «les Furies vengeresses», «le livide Orchus». Orchus est une divinité infernale, il personnifie ici la mort.

68. «Par une chute plus grave »; Secretum I, 60 : «gravior casus »; " une chute plus importante».

69. "Ma barque fissurée»; Secretum I, 62: «tremulam cimbam fatiscentemque et rimosam»; «ma barque ballottée, épuisée, et lézardée».

70. «(Un rocher) englouti parfois battu par des vagues bouillonnantes»; Secretum I, 62 : «ventis obluctantibus per tumidos fluctus ago "; «je me dirige à travers les vagues bouillonnantes aux prises avec les vents contraires».

71. "Nous avons vécu dans les flots, puissions- nous mourir au port»; Secretum I, 62: "qui in pelago vixerim moriturus in portu»; "moi qui ai vécu en haute mer, (je voudrais) mourir au port". 
De l'examen de ces renvois nous pouvons tirer des conclusions qui vont dans le même sens que celui des citations explicites, en exacerbant encore la précellence des auteurs antiques, même dans la bouche d'Augustinus, qui semble alors avoir un discours «contradictoire».

Le corpus est élargi aux poètes élégiaques comme Stace et Properce, aux Bucoliques de Virgile, et à d'autres œuvres philosophiques de Cicéron.

C'est encore Augustinus qui a le plus recours à ces auteurs.

En revanche, la hiérarchie des auteurs n'est plus la même sur la totalité, ni pour les deux personnages. Cicéron et Sénèque deviennent les auteurs les plus évoqués, à égalité, et deux fois plus chacun que Virgile. Pour Franciscus l'auteur le plus cité devient Cicéron pour moitié, suivi par Virgile et Horace à égalité et les autres poètes, dont les poètes élégiaques, auxquels il emprunte le vocabulaire de la labilité, et notamment Ovide (Tristes et Pontiques). Augustinus quant à lui doit son discours pour moitié à Sénèque, suivi par Cicéron, et trois poètes, beaucoup moins cependant que pour les citations explicites.

$\begin{array}{ll}\text { Source Euvre } & \text { NB } \\ \text { Cicéron } & 12 \\ \text { De finibus III, 7, 26 } & 1 \\ \text { De Oratore, proem. } & 1 \\ \text { De Senectute XIX, 67 } & 1 \\ \text { De Senectute XIX, 71 } & 1 \\ \text { De Senectute XX, 74 } & 1 \\ \text { Tusculanes III, 10, 23 } & 1 \\ \text { Tusculanes III, 11, 24 } & 1 \\ \text { Tusculanes IV, 17, 37 } & 1 \\ \text { Tusculanes V, 10, 29 } & 1 \\ \text { Tusculanes V, 29, 83 } & 1 \\ \text { Tusculanes V, 8, 23 } & 1 \\ \text { Tusculanes V, 9, 25 } & 1 \\ \text { Horace } & \\ \text { Odes I, 22, } 1 & 4 \\ \text { Odes II, 2, 2 } & 1 \\ \end{array}$

72. "Pour tous les autres troubles, qui se divisent en quatre genres, et plusieurs espèces"; Secretum I, 64: "discerno clarissime quadripartitam animi passionem »; "je vois très clairement que la passion de l'âme se divise en quatre». 
Citations et sources antiques dans le Secretum de Pétrarque

Odes III, 10, 10

Odes IV, 13, 10-11

Ovide

Tristes I, 11, 23

Tristes III, 416

Properce

III, 17,6

Sénèque

Lettres à Lucilius LXX, 18

Lettres à Lucilius XXII, 14

Lettres à Lucilius CXVI, 8

Lettres à Lucilius LXVII, 4

Lettres à Lucilius LXX, 15

Lettres à Lucilius LXXVII, 11

Lettres à Lucilius VI, 1-2

Lettres à Lucilius VI, 6

Lettres à Lucilius VII, 12

Lettres à Lucilius XCII, 10

Lettres à Lucilius XIX, 2

Stace

Thébaïde XI, 252

Virgile

Bucoliques II, 24

Enéide I, 315

Enéide I, 324

Énéide V, 125

Enéide VI, 273-296

Enéide VI, 29

1

2

1

1

1

1

1

1

1

6

1

1

1

1

1

1

Total

LOCUTEUR

SOURCE

NB

Augustinus

Cicéron

Horace

Ovide

Sénèque

\section{Stace}

Virgile 
Franciscus

Cicéron 9

Horace 2

Ovide 1

Properce 1

Sénèque 3

Virgile 2

La vérité

Horace 1

Virgile 1

Total

Au terme de ces examens approfondis des citations antiques et des renvois à des auteurs latins dans le prologue et le livre I du Secretum, nous avons voulu montrer comment s'exprimait l'amour de Pétrarque pour ses chers auteurs latins. Il est intéressant de voir qu' en réalité, dans le discours "officiel», Augustinus, qui lui reproche cette attitude et cette préférence, n'a pas le dernier mot, voire même se contredit, en citant plus souvent que Franciscus les auteurs anciens, et notamment Virgile, le poète que Pétrarque voulait égaler. En revanche, dans le discours "souterrain", celui où les deux personnages s'affrontent, chacun reprend son rôle, celui de Sénèque pour Augustinus et celui de Cicéron pour Franciscus. Ceci est le début d'une controverse sur ces deux philosophes romains, dans leur conception de la vie, de la littérature, du style, etc. qui fleurira à la Renaissance, dont Pétrarque là encore est un précurseur ${ }^{73}$. Virgile reste cependant le héros secret du Secretum... Dans une lettre écrite vers l'âge de cinquante-cinq ans, Pétrarque ne dira-t-il pas qu'il aime Virgile et Cicéron d'une affection qui dépasse celle qu'on peut avoir pour un être vivant? ${ }^{74}$

73. Voir Christian Mouchel, Cicéron et Sénèque dans la Rhétorique de la Renaissance, Marburg, 1990.

74. Familiares XXII, 10 